\title{
Fibroepithelial Polyp at the Bladder Neck Presenting with Gross Hematuria in a 5-Year-Old Boy
}

\author{
Avni Merter Keçeli, MD, ${ }^{1}$ Muhammet İrfan Dönmez, MD, FEBU, FEAPU, ${ }^{2}$ and Ayşe Nur Uğur Kılınç, MD ${ }^{3}$
}

\begin{abstract}
Background: In any age group, gross hematuria is an alarming symptom. A variety of diseases, including urinary tract tumors, urinary tract infections, glomerulonephritis, urinary stone disease, and urinary trauma, can cause gross hematuria. Neoplasms of the urinary tract are definitely the most bothersome among the differential diagnoses. Fibroepithelial polyps (FEPs), in contrast, are mesodermal-originated rare and benign tumors that can be observed in the urinary tract. In this case report, we present a child with an FEP at the bladder neck that was referred because of gross hematuria.

Case Presentation: A 5-year-old boy presented with intermittent gross hematuria for 2 weeks. Ultrasonography showed a $2 \times 1 \mathrm{~cm}$ mucosal surface-borne polypoid lesion at the trigon. Patient underwent cystoscopic evaluation that revealed a polypoid mass located at the bladder neck; however, there were no signs of bladder outlet obstruction in the bladder. Transurethral resection revealed an FEP.

Conclusion: Hematuria should be evaluated carefully not only in adults but also in children. FEPs in bladder can mimic bladder carcinoma clinically and radiologically. It should be kept in mind in the differential diagnosis of bladder lesions on imaging modalities in pediatric population.
\end{abstract}

Keywords: fibroepithelial polyp, pediatric, hematuria

\section{Introduction and Background}

$\mathbf{H}$ EMATURIA IS A FINDING that can be observed in nephrologic and/or urologic disorders in both pediatric and adult population. Urinary tract tumors are an important cause of hematuria. ${ }^{1}$ Fibroepithelial polyps (FEPs) are quite rare benign tumors with mesodermal origin. FEPs are most commonly located in the renal pelvis, less frequently in the bladder and distal ureter. ${ }^{2}$ FEPs are rarely found in the bladder, especially in the childhood. ${ }^{3}$ In this report, we present a 5-year-old boy with FEP at the bladder neck causing intermittent gross hematuria rather than bladder outlet obstruction.

\section{Presentation of Case}

A 5-year-old boy referred to pediatric urology clinic with intermittent total gross hematuria without clots for 2 weeks. He had no other symptoms such as pain, fever, or vomiting. Urine analysis was normal that revealed no proteinuria. His physical examination was unremarkable with no periorbital edema. His blood pressure was within normal range for age. Urinary ultrasonography (US) revealed normal pelvicaliceal system with no ureteral dilation but a $2 \times 1 \mathrm{~cm}$ mucosal surface-borne polypoid lesion at the trigon of the bladder (Fig. 1A). Bladder wall thickness was normal. Perivesical fat plans could be distinguished (Fig. 1B). In color and power Doppler US, vascularization was not observed in the lesion (Fig. 1C). Also, there were no stones in the urinary tract.

Complete blood count, serum creatinine, c3 and c4 levels, and coagulation tests were within normal range. Cystoscopy was performed to evaluate the lesion. A pedunculated polypoid mass arising from the bladder neck was detected (Supplementary Video S1). Subsequently, it was excised using a pediatric monopolar resectoscope (Fig. 1D). Urethral catheter was kept in situ for 2 days. Histopathologic examination revealed a polyp covered with urothelial epithelium that had loose fibrovascular stroma (Fig. 2A). Urothelial epithelium was mature and there were no signs of atypia and/ or dysplasia (Fig. 2B). Thus, the specimen was diagnosed as a benign FEP.

\section{Discussion and Literature Review}

FEPs are mesodermal benign tumors, which are rarely found in infants and children. The etiology of FEP is still unknown. These lesions are often seen in the renal pelvis and distal ureter; they are rarely located in the bladder and

Departments of ${ }^{1}$ Radiology, Pediatric Radiology, ${ }^{2}$ Urology, Pediatric Urology, and ${ }^{3}$ Pathology, Konya Training and Research Hospital, Konya, Turkey. 


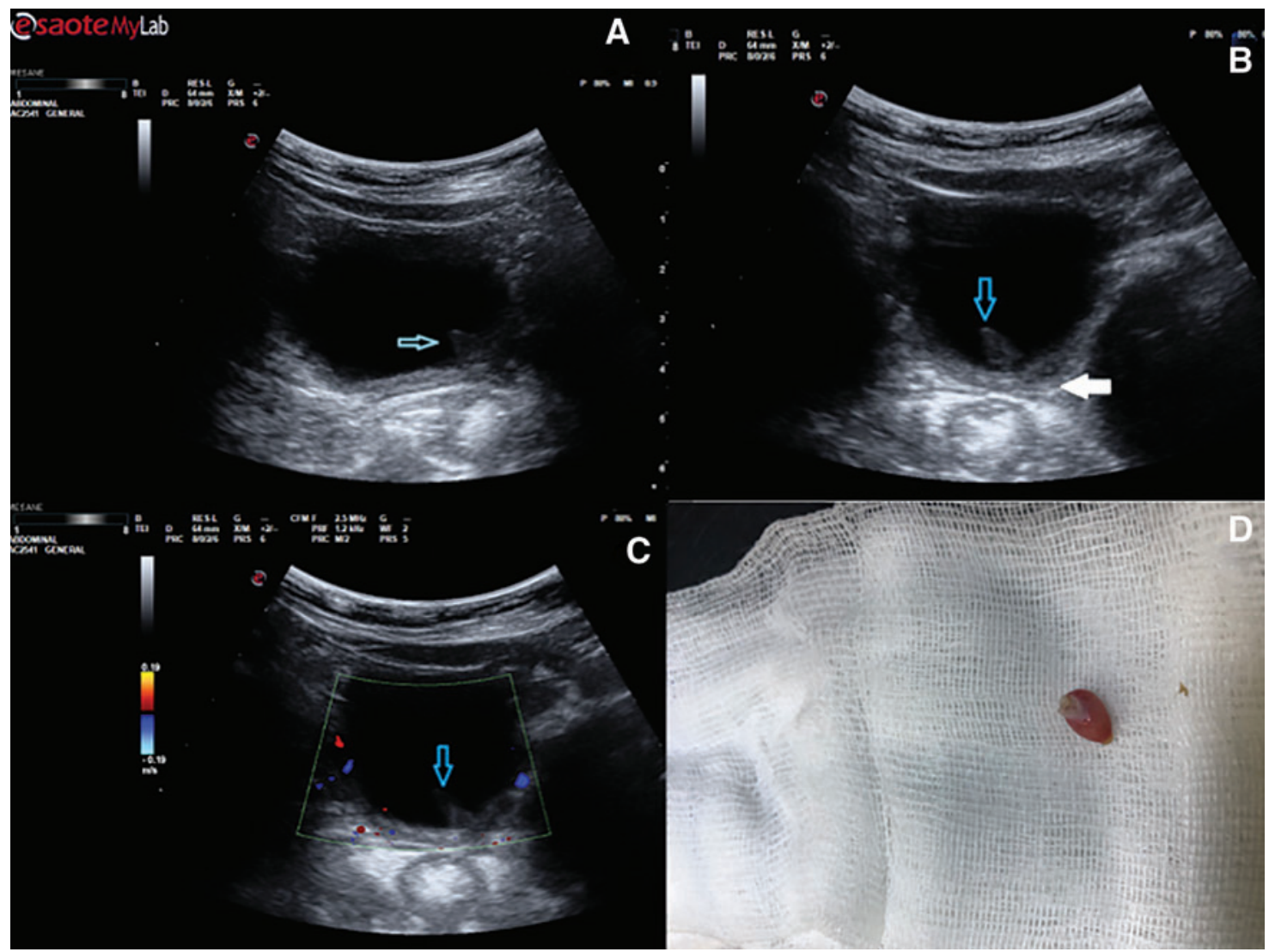

FIG. 1. (A) Axial plane sonogram image at pelvic level, mucosal surface-borne polypoid lesion in the bladder at the trigon level (arrow). (B) Axial plane sonogram image at pelvic level, the polypoid lesion (open arrow) is seen. Bladder wall thickness is normal, perivesical fat planes are clear (solid arrow). There is no sign of invasion. (C) Axial plane sonogram image at pelvic level, in color Doppler US, vascularization is not seen in the polypoid lesion (arrow). (D) The polyp specimen, right after en bloc resection. US, ultrasonography.

proximal ureter. In addition to hematuria, they may cause urinary tract obstruction (hydronephrosis) and can cause severe pain when torsioned. ${ }^{4}$ The ureteral FEPs may advance into the bladder cavity mimicking a bladder stone. They are often congenital in children, whereas in adults they may be caused by inflammation, chronic irritation, and infection. Previous studies have reported that FEPs manifest in children with a mean age of 9 years and have a strong male predominance. Bladder FEPs do not have specific radiologic features to distinguish from other pathologic bladder lesions. Vascularity of the lesion may be beneficial for differentiation, which is not present in FEPs.

The most common finding of FEPs is hematuria where US is the first imaging modality used for diagnosis. FEPs that form filling defects in the urinary system can be demonstrated by intravenous pyelography, retrograde ureteropyelography, CT, or Magnetic Resonance urography. ${ }^{4}$ Often, radiologic imaging may not be sufficient for radiologic diagnosis of
FIG. 2. (A) The polyp covered with urothelial epithelium and has loose fibrovascular stroma transitional epithelium covering the fibrovascular stroma. (HE, $40 \times$ ). (B) Histopathologic examination of the polyp, there is no sign of atypia and/or dysplasia (HE, 40×). HE, hematoxylin eosin.

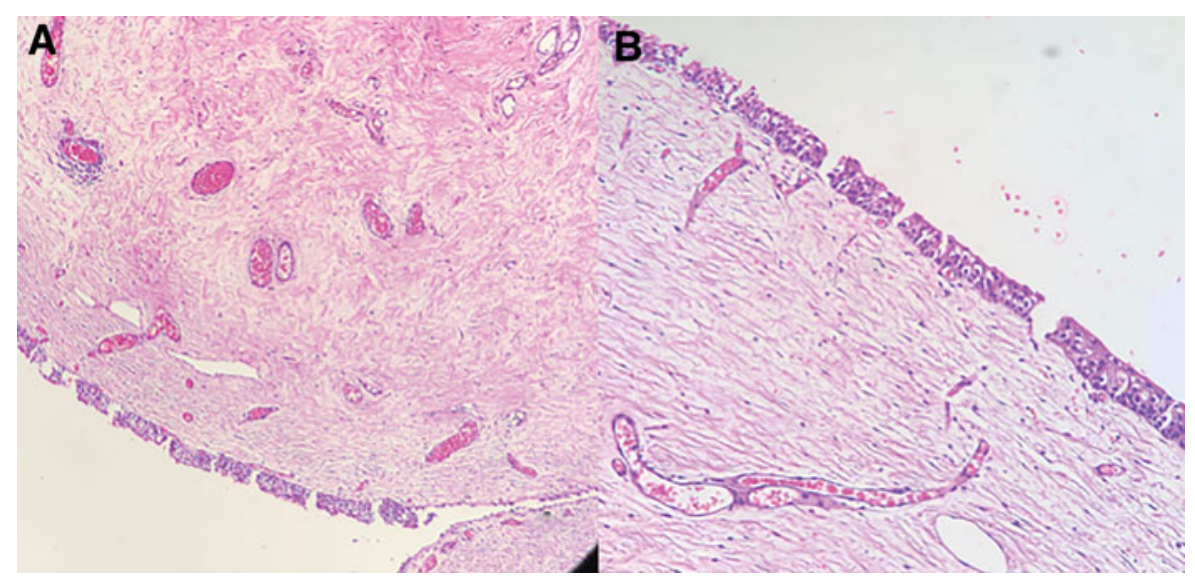


FEPs and endoscopic intervention is required. However, urinary US was effective in observing the lesion in this case. Therefore, no further imaging was obtained to reduce radiation exposure for the child. Needless to say, US would not be adequate for staging, and a CT urography would be required if the pathology analysis of the mass turned out to be an invasive malignant tumor.

During cystourethroscopy, it is easy to differentiate the smooth surface appearance of FEPs from irregular and papillary urothelial carcinomas. They are often seen as fingerlike protrusions arising from the mucosa attached to a narrow base. However, they may also be sessile. Histopathologic evaluation provides the definitive diagnosis. The lesions are mostly solitary while in a retrospective screening; multiple polyps were detected in $27 \%$ of the cases.

In pathologic examination with hematoxylin eosin staining, FEPs are composed of papillary leaves lined by a normal appearing urothelium without cellular atypia. Some of them may exhibit ulceration.

A large number of ureteral FEPs have been reported. Almost all of these lesions show signs of urinary tract obstruction. However, there are only a few reports of benign fibroepithelial bladder polyps in the literature. FEPs located in the bladder especially at the bladder neck may cause bladder outlet obstruction.

The risk of progression to an invasive neoplasm of a benign FEP is less likely with only a few cases reported in literature. However, complete resection is the optimal method to avoid progression and recurrence. Using electrocautery, as well as laser, is the most widely used method for resection.

\section{Conclusion}

Hematuria should be evaluated carefully not only in adults but also in children. FEP in bladder can mimic bladder carcinoma clinically and radiologically. FEP should be kept in mind in the differential diagnosis of bladder lesions in the pediatric population.

\section{Disclosure Statement}

No competing financial interests exist.

\section{Funding Information}

No funding was received for this article.

\section{Supplementary Material}

Supplementary Video S1

\section{References}

1. Meyers KE. Evaluation of hematuria in children. Urol Clin North Am 2004;31:559-573.

2. Liddell RM, Weinberger E, Schofield DE, Pelman RS. Fibroepithelial polyp of the ureter in a child. AJR Am J Roentgenol 1991;157:1273-1274.

3. Zachariou AG, Manoliadis IN, Kalogianni PA, Karagiannis GK, Georgantzis DJ. A rare case of bladder fibroepithelial polyp in childhood. Arch Ital Urol Androl 2005;77:118-120.

4. Shelmerdine SC, Lorenzo AJ, Gupta AA, Chavhan GB. Pearls and pitfalls in diagnosing pediatric urinary bladder masses. Radiographics 2017;37:1872-1891.

Address correspondence to: Muhammet İrfan Dönmez, MD, FEBU, FEAPU

Urology, Pediatric Urology Konya Training and Research Hospital Hacışaban Mahallesi, Yeni Meram Cd. No. 97

Meram 42090

Konya

Turkey

E-mail: m_irfan83@yahoo.com
Cite this article as: Keçeli AM, Dönmez Mİ, Uğur Kılınç AN (2020) Fibroepithelial polyp at the bladder neck presenting with gross hematuria in a 5-year-old boy, Journal of Endourology Case Reports 6:3, 107-109, DOI: $10.1089 /$ cren.2019.0125. 